\title{
Effective Following Patterns Mining Scheme for the Movements of Objects
}

\author{
Li Chen, Lianggui Liu*, Bingxian Chen, Huiling Jia and Yu Zhang \\ School of Information Science and Technology, Zhejiang Sci-Tech University, \\ Hangzhou 310018, China \\ kunjiande@163.com
}

\begin{abstract}
Technological advances of location aware devices allow us to locate and track the moving objects accurately and in more complex environments. In order to derive valuable knowledge embedded in data from databases, many domestic and foreign scholars have done a lot of work. In this paper, we review the progress of patterns mining mainly in frequent pattern, periodic pattern and following pattern. And we provide a general perspective for studies on different patterns mining by reviewing and comparing the methods and algorithms in detail, providing a quick understanding of research to the worker and giving effective following patterns mining scheme for the movements of objects. The study on patterns mining in movements not only has a direct bearing on human life but also play an important part in environment protection, traffic and transportation, privacy and security and so on. So, the rest of this paper will present these specific applications and analyzed it in theory.
\end{abstract}

Keywords: Patterns mining, Frequent pattern, Periodic Pattern, Following pattern

\section{Introduction}

The increasing use of location-aware devices has led to an increasing availability of mobility data, such as trajectory data of moving objects [1, 2], traffic data [3], climate data [4], and animal migration data [5-7]. The availability of such data on devices will inevitably engender the study of patterns mining at an unprecedented scale both in terms of the areas covered by the moving objects and also the number of individuals involved in the study. Trajectory data of moving objects, simply, is the location of the sequence by the number of sampling points in accordance with the chronological order; from a structural point of view, is a multi-dimensional data composed by the object identification, sampling location, sampling time and other information. These trajectory data as historical activity data of moving objects, in some extent, show the internal and external feature of moving object, such as properties, state, active characteristics. How to mine patterns of moving objects efficiently, it is great help for studying moving objects.

In recent years, with the rapid development of patterns mining of moving objects, the relevant pattern mining method is also increasing. So far the typical trajectory pattern mining methods can be roughly divided into two categories: mining methods based on clustering [8-10] and pattern mining method based on frequent sequence [11-13]. Also, the study on mining patterns for moving objects has made some progress at home and abroad. In 2001, Tsoukatos et al. [14], who first proposed patterns mining in spatiotemporal datasets, and proposed a new trajectory pattern mining algorithm DFS_JMINE (Depth First Search Mine).This algorithm has the advantage that the amount of space required is minimal. In 2005, Maumoulis et al. [15] a newly proposed substring tree to accelerate search for longer patterns. Giaimott et al. [16] proposed a new pattern

Lianggui Liu is the corresponding author. 
named T-patterns and several different methods. Next, an efficient graph-based mining (GBM) algorithm [17] was proposed. With the deepening of the research, tree-based interaction mining algorithms [18] are designed to analyze the structures of the trees and to extract interaction flow patterns. Recently, Chih-Chieh et al. [19] proposed a new trajectory pattern mining framework, namely Clustering and Aggregating Clues of Trajectories (CACT), for discovering trajectory routes that represent the frequent movement behaviors of a user.

Nowadays, we study a lot of categories of patterns that can be discovered from a single trajectory or a group of trajectories. The main patterns are periodic patterns, sequential patterns, following patterns, frequent patterns. Also, researchers in patterns mining have made important contributions by developing methods and tools to solve specific application problems. For instance, road traffic monitoring [20], epidemic prevention [21], travel route recommend [22, 23], natural disaster warning [24, 25], environmental protection [26, 27], urban planning [28], animal behavior analysis [29].

This paper will focus on the patterns mining based on moving objects, with a target on researches that aim to give an integrated view of progress in patterns mining. The remainder of this paper is organized as follows: Section 2 focuses on detail and results in mining various patterns about frequent patterns, periodic patterns, following patterns. In addition, Section 3 will introduce the specific application in each field. In Section 4, we briefly conclude this paper.

\section{Patterns Mining}

All in real life, the track of moving objects is known, but the potential activity regularity is unknown. However, these unknown activity patterns and activity rule for researchers are more important. In general, the moving objects, can reflect the characteristics and laws of activity objects, and these laws are made up of several independent objects together, all the activities of the object mutual combination or affecting the group characteristics and laws of life. With the aid of the means and methods of data mining, we will get the object's movement rule and behavior patterns from trivial confused mobile object trajectory data. We can find frequently repeated path in a mobile dataset, which can help the researchers to complete analysis and prediction about moving objects, and can be applied to business, tourism and management of urban traffic aspects of decision-making.

For the sake of analyzing the massive-scale moving object data flexible and scalable, a system, named MoveMine, is designed by Li et al. [30], which can sophisticate moving object data mining by integrating several attractive functions including moving object pattern mining. This system has a major functional module describes the result of interaction several patterns, generally have swarm pattern and following pattern, periodic pattern. Through in-depth analysis of these patterns, can discover the regularity of the activities of moving objects help to analyze and forecast object activity as well as the direction in the future, can also be used to track the species, and pollutants transportation network planning. However, this system does not include recent methods on relationship pattern mining. Thus, in 2014, Wu et al. [7] propose MoveMine 2.0 by adding substantial new methods in mining dynamic relationship patterns. MoveMine 2.0 focuses on two types of pairwise relationship patterns that are attraction/avoidance relationship and following pattern. In subsequent chapters of this section will continue to introduce a variety of patterns mining in detail.

\subsection{Frequent Patterns}

Moving objects frequent patterns are the sequences that recur in the moving objects. In the process of frequent pattern mining for moving objects data, consist of three main steps: (1) extracting a region of interest of moving objects; (2) the moving objects data 
sets are converted to set the region of interest, and the interest region has a certain geographical area, area representative point, time and other attributes; (3) the use of a moving object time frequent pattern mining algorithms to extract frequent patterns of moving objects.

Moving Objects frequent patterns mining is mainly on a number of tracks sequence mining, the results will not represent specific sequences of all tracks. Thus, depending on defined conditions, such as setting the transition time is not exactly the same, and sometimes choose a track sequence represents the frequent pattern mining results, and sometimes will select the other tracks sequence represents the frequent pattern mining results, but the results are mining all tracks sequence represents a trend in the behavior of the activities defined conditions.

At present, the moving objects algorithm for mining frequent patterns has been perfect. Frequent pattern mining of moving objects can be formalized as a frequent sequence mining [31], but the moving objects data includes location dimension, time dimension and semantic dimension, so simply using the traditional sequence mining methods cannot effectively solve the problem of frequent pattern mining. Giaimott et al. [16] improved the traditional frequent sequence mining method, and proposed mining a frequent sequence which configuration by the region of interest (Region of interest, ROI). Simultaneously, they proposed a new pattern, called a trajectory pattern (T-Patterns), represents a set of individual trajectories that share the property of visiting the same sequence of places with similar travel times. This pattern mining method fully integrated time and space property, it had been applied to frequent pattern mining representative. Among them, the space property represents the geographic area that the track has stay for a time, and time attributes behavioral activities on behalf of a moving object in a certain region of space travel time, including a series of sequential access. On the basis of the method, Giannotti et al., who have conducted in-depth research, proposed a tree structure method of mining frequent patterns of moving objects, many T-Patterns of the results of this method have been associated with each other, and then based on the tree structure gives a strong theoretical basis for the future location prediction. They put forward three different algorithms for mining frequent patterns: (1) in the data preprocessing phase, the point where the track sequence is converted into a sequence from the region of interest; (2) according to the different forms of spatial discrete, preset region of interest were two forms of the region of interest and popular areas of interest. The first two pattern mining algorithms are realized based on these two areas of interest; (3) the third algorithm 3 mainly considers how to combine the spatial and temporal dimensions of pattern mining. According to local area density variation, construct a new mining algorithm with incremental approach to discover region of interest. Experimental results show that this method can be identified more accurately patterns.

The process of this patterns, need to convert the track position sequence, and therefore it is more suitable for geospatial systems with practical meaning, as for the need to analyze the frequent pattern of entire trajectory does not apply. This case needs to compare the degree of similarity between two trajectories, and using the method of clustering to together the same movement patterns. The distance between the tracks can be divided into Euclidean distance, the minimum bounding distance, DTW distance and the longest common subsequence and so on. Coordinate point distance is on track as a measure of the standard, taking into account the time between the coordinate points. In [32] calculates similarities between trajectories by the direction of movement of moving objects, speed and other attributes.

Hung et al. [19] propose a new trajectory pattern mining framework, namely Clustering and Aggregating Clues of Trajectories (CACT), for discovering the frequent movement behaviors of a user. This framework notices existing clustering techniques do not deal with silent durations. Thus, they formulated a clue-aware trajectory similarity and a clue-aware clustering algorithm to cluster similar trajectories into groups. For each 
group, these trajectories aggregated by CACT used to identify hot regions and to discover these frequent patterns.

In order to analyze and study the case of the most frequent path selection of the majority pedestrian, in 2013, Li et al. [33] studied the query problem of the most frequent path based on the time period. In order to avoid the path number of edges and the frequent path acting on mining results, they chose the sequence to describe the passing frequency, no count the passing frequency based on the scalar value function. The concrete steps: (1) structure a diagram containing the edge weights footprint at a specified time interval, also use footprint indexes to improve the computational efficiency of structuring the footprint chart; (2) use dynamic programming algorithm solve the problem of the path query, then take the improved Bellman-Ford algorithm for final problem solving.

For the purpose of finding minimum representative pattern sets in an enormous number of the generated patterns for further effective analysis. Liu et al. [34] have proposed two algorithms, MinRPset and FlexRPset. Both algorithms first mine frequent patterns, and then find representative patterns in a post-processing step, while MinRPset is very spaceconsuming and time-consuming on some dense datasets when the number of frequent closed patterns is large. FlexRPset provides one extra parameter $\mathrm{K}$ to allow users to make a trade-off between result size and efficiency to solve this problem. Also, MinRPset and FlexRPset have some drawbacks. In the follow-up study, they need to be solved. But, in real life, only a handful of frequent patterns can be converted into practical application. To solve this problem, many algorithms have proposed, such as top-k frequent closed pattern mining, FAE and VTK algorithms and NTK algorithm for mining top-rank-k frequent patterns based on the idea of PPC-tree. As NTK must generate and test all candidates in each loop of the algorithm. Therefore, Quyen [35] presents an improved method, iNTK, for mining top-rank-k frequent patterns. This method uses N-list structure with an improved $\mathrm{N}$-list intersection function to reduce the run-time and memoryconsuming and employs the subsume index concept to directly mine frequent.

Large number of missing, incomplete, inaccurate, and duplicated records exists in the RFID-enabled logistics data, though they carry rich information that could be used for further and advanced decision-makings. In [36] introduced a Big Data approach for mining the invaluable trajectory knowledge from such data. Specifically, several key procedures are proposed: a RFID-Cuboid cleansing algorithm is presented for detecting and removing the noise data from the logistics dataset, a RFID-Cuboid compression algorithm is demonstrated for reducing the storage space and enhancing information granularity, and a RFID-Cuboid classification algorithm is reported for clustering the cuboids according to the practical applications/considerations.

\subsection{Periodic Patterns}

Frequent patterns mining is primarily used to detect frequent activities and the path that frequently accessed in moving objects. While the periodic patterns mining is focused on the feature to find the time characteristics of repetitive activities on the basis of the moving objects activities. Existing periodic patterns mining is mainly divided into full periodic patterns mining, partial periodic patterns mining, asynchronous periodic patterns mining, and association periodic mining. The approaches used in the existing periodic pattern mining algorithms, have several limitations. For instance, suffix tree based algorithm [37], that is, if we use the suffix tree to generate patterns and detect periodicity, we will fail to generate some flexible and interesting patterns. Then, Manziba et al. [38] proposed new algorithm has overcome this limitation. Recently, there are many existing algorithms about periodic patterns mining.

Full periodic patterns mining can be seen as a continuous process throughout the cycle, means that the sequence of each object (or item) affects the cycle sequence. In [39], they first proposed the full periodic patterns mining and an efficient algorithm: the interleaved algorithm. They utilized the characteristics of full periodic patterns to prune the irrelevant 
data and make the algorithm very efficient through a series of experiments. It also means that this approach cannot be used to solve the more general problem of partial periodic patterns mining. Elfeky et al. [40] considered these unknown or obscure periods in time series databases discovered that is part of the mining process. Thus they developed an algorithm mining periodic patterns by an adapted definition of convolution. The algorithm is computationally efficient as it scan the data only once. Owing to the inferior quality of mining patterns Kim et al. [41] proposed hash filtering and quantity sampling techniques that significantly improve the performance of the naive extensions. Mining periodic behavior for moving objects could be complicated.

Partial periodic patterns mining compared with full periodic patterns mining, the main difference lies in the time series for each pattern or each item is periodic in the entire process behavior. Partial periodic patterns only concerned with period features of time series at some local points, but not all time points. The main concept of the partial periodic pattern mining was first proposed by Han et al. [42], it was the mixture of periodic events and non-periodic events in the same period. Afterward, many published studies about partial periodic pattern mining have applied the concept of the max-subpattern hit sets.

A periodic time series may be interrupted by noise data in time series. Faced with this problem, many scholars put forward the concept of asynchronous periodic patterns, and proposed asynchronous periodic pattern mining algorithm. These authors in [43] proposes an asynchronous periodic sequential pattern mining model, noise can be tolerated in this model. The model was depicted: first, judge whether a sequence appears repeatedly. Then, determine whether the time interval in periodical sequence is random noise or the change of system behavior. Lastly, the time periods sequence appears periodically would be jointed to get the maximum periodic time range. Also, the patterngrowth mining algorithm employs a dividing and rule method with multiple minimum item supports.

Currently the most common pattern is association periodic mining, which is used for mining internal relevance in periodic items and relevance of the time of occurrence. To address the problem of mining periodic behaviors for moving objects, these authors in [44] proposed a two-stage algorithm, Periodica. In the first stage, periods are detected through reference spots using Fourier transform and autocorrelation. In the second stage, periodic behaviors are statistically summarized using hierarchical clustering method. This algorithm successfully solves two problems: how to detect the periods in complex movement, and how to mine periodic movement behaviors.

Based on solving the problem of mining periodic-frequent patterns in very large databases, Kiran et al. [45] put forward the PFP-growth ${ }^{++}$algorithm, Specific as follows, first, the database would be compressed into a tree structure. Then, mining the complete set of periodic-frequent patterns by exploring the PF-tree ${ }^{++}$recursively.

\subsection{Following Patterns}

Following patterns mining is widely used in discovering the objects with the same pattern or similar pattern and in the relationship of different objects. Group Concurrence and flock [46] are considered the dimensions shape of following patterns. The definition of flock pattern is broad and the lack the necessary restrictions, will mine meaningless following patterns. In order to overcome the defects of flock pattern, Li et al. [47] build restrictions on the spatial dimensions for flock pattern. Therefore, more accurate and practical significance following patterns could be mined. In 2005,Panos Kalnis et al. [48] put forward the concept of Moving Cluster and for the first time introduced the concept of density associative in the pattern mining, which can effectively cluster each time or time slice target groups and can find following pattern in irregular groups. The restriction is a prototype area that the radius size is set artificially. If the radius is set over the General Assembly would reduce the efficiency of mining and digging out no realistic results, and 
set the radius is too small will cause the loss of the mining results due to moving objects and lead mining inaccurate results.

In 2006, Wang et al. [49] proposed two mining algorithm: AGP and VG-growth and data compression algorithm based on these mining algorithms. Also, Mattias et al. [50] provided a formal description of the pattern 'leadership' and subsequently algorithms for its efficient detection. 'Leadership' describes the event or process of one individual in front leading the movement of a group. However, the drawback of the given definition of leadership is that a leader has to be in the front region of all followers. In 2008, Hoyoung et al. [51,52] put forward the concept of Convoy and the corresponding mining algorithm, which overcome the drawback of Moving Cluster ignore the similarity comparison of historical data and current data. Also, the CuTS algorithm uses Douglas-Peucker (DP) algorithm and its improved algorithm to simplify the original temporal trajectory, thus improving the algorithm time efficiency. In 2010, Li et al. [53] proposed the concept of swarm similar to Dynamic convoy [54] and also put forward the corresponding mining algorithms S3(Simple Slice-by-Slice), The Object Growth Method, to further improve the efficiency of mining algorithm of time, and digging out before neglected pattern. Similar to the concept of following pattern, Fernando [55] put forward the method to discover the chasing behavior. They present an algorithm to find the sub-trajectories that contain a chasing pattern. In order to set the appropriate parameters, they used the time dimension to make the best.

In 2012, Zheng et al. [56] put forward mining algorithm based on travelling buddy ,which was on efficiency as there have been significantly improved, but not for the current big data environment to optimize performance. When faced with large data, the algorithm and has some other time-consuming algorithms would be unacceptable. The challenges in mining the following patterns are: (1) the following time lag is usually unknown and varying; (2) the trajectories of the follower and leader are not identical; and (3) the relationship is subtle and only occurs in a short period of time. In order to addresses all these challenges, Li et al. [57] proposed a simple but practical method that requires only two intuitive parameters $\left(\mathrm{d}_{\max }, \mathrm{l}_{\max }\right)$. They first transformed the problem into the well-known local sequence alignment (LSA) problem. Then, they used the SmithWaterman algorithm for LSA to find intervals with most matching pairs. To solve the problem efficiently, it could be transformed into the well-known Maximum Sum Segment Problem, for which the optimal solution can be found in linear time. While the method has been focused on mining following patterns between two moving objects, would not be practical in real life. Ulanbek et al. [58] used the FPM approach for discovering moving flock patterns in large spatiotemporal data sets. The FPM approach showed to be useful to deal with the problems found in the BFE (Basic Flock Evaluation) algorithm for data sets with a large numbers of trajectories.

\subsection{Other Patterns}

Relationships between two moving objects based on their spatiotemporal interactions may be attraction or avoidance. It is a pair of interesting relationship that detected by counting the frequency of objects being spatially close. In previous times, Laube et al.[59] proposed the method of REMO pattern detection to match more patterns, such as leadership, recurrence, flock, encounter, convergence. These patterns differ from each other based on movement, direction, and location. Specifically as follows: The leadership pattern means that at least one of the moving objects is leading the direction that at least in a certain period of time and have at least $m$ subtrajectories within a circular region of radius $r$ that move in the same direction. Reccurrence patterns occur when at least $m$ entities visit a circular region at least $k$ times. A flock pattern has at least $\mathrm{m}$ subtrajectories within a region of radius $r$ that move in the same direction during a certain time interval. Encounter is the pattern characterized by at least $\mathrm{m}$ subtrajectories that are concurrently 
inside the same circular region of radius $r$, assuming they move with the same speed and direction.

Li et al. [60] proposed to use a popular non-parametric approach, permutation test, to performing hypothesis tests and constructing confidence intervals. Firstly, they computed the meeting frequency and significance value which represent the degree of the relationship of moving objects. Then, the counting algorithms are based on the following basic Monte Carlo scheme is proposed. Considering the calculation efficiency, they proposed two pruning techniques below that can greatly speed up this test.

In the event group object, there is always an individual object is responsible for security of the entire population, forming the convoy pattern. These are many algorithms for mining convoy pattern, but do not scale to real-life dataset sizes. Therefore, Faisal et al. [61] used distributed algorithm to settle this problem. They analyzed different data partitioning strategies for mining convoy patterns in a distributed shared-nothing architecture based on these properties. Also the distributed algorithm for convoy pattern mining can be divided into three stages: partitioning, local convoy pattern mining and merging to produce the global result.

Existing algorithms about mining moving object clusters on the consecutiveness of timestamps may result in the loss of interesting patterns, or risks discovering noisy patterns. Li et al. [62] put forward a new type of pattern called the platoon pattern. Compared to an existing method for discovering moving object clusters, their algorithm won in running time. The platoon pattern is proposed based on group pattern, having many applications in real life. Unlike the globally consecutive timestamp constraint of the convoy pattern, a platoon only requires that the timestamps are locally consecutive. Also, the algorithm employs four types of pruning rules to discover the set of closed platoons.

With the rise of the Internet and smart phones, human life has changed tremendously by monitoring human activities in real time. Studies on long- term mobility data could be important for urban planning, transportation and prevention of human diseases. For that, Roberto et al. [63] come forward a new pattern named correlation patterns. The concept of C-pattern is that a set of regions occur in the specific order different from their common behavior, not happen at the same time or at different times. They did this in three steps: first, detect the locations and times where relevant variations of population take place; second, infer from them more complex patterns that link regions where variations tend to appear together or in some constant sequence; finally, navigate the discovered patterns along the spatial and temporal dimensions, and enrich patterns with additional information (derived from raw data) to help their interpretation. In the process of mining patterns, C-SPAM algorithm which extends SPAM algorithm was used.

\section{Applications and Future Works}

Moving objects patterns mining have a range of real-world applications. These data from moving objects has been used for analyzing and inferring an individual's behavior patterns and lifestyles which in turn can reveal information about and implications. A great many systems and applications are designed for monitoring patient's health and analyzing people's everyday lifestyles. Qiu et al. [64] investigated a computation framework based on four layers of progressive analysis, which would will facilitate the discovery of an individual's activities and behavior patterns from their GPS traces. Specifically, they are data pre-processing layer, trajectory identification layer, semantic annotation layer and semantic trajectory analytics layer. Also, these applications play an active role in everyday human life, criminal case, transportation, or monitoring animal behavior and so on. Details as follows:

- Daily Life: Owing to the pervasiveness of GPS-equipped mobile devices today, the locations of users such as people, vehicle can be easily located. By analyzing the trajectory data, we can observe some hot spots and discover the frequent patterns or 
periodic patterns. This information can be used to the APP of mobile phone for tourist attractions, navigation route recommendation or route design.

- Criminal Case: We often encounter police manhunt on the road or track down criminals. Considering the number of tracking records, if know the following patterns of these moving objects, it would be extremely easily for people to inspect them.

- Transportation: The identification of common routes among vehicles may lead to more effective traffic control and the early discovery of truck patterns may assist traffic planning to avoid congestion. Zhang et al. [65] put forward the algorithm iBAT / iBOAT analyzing taxi trajectory data to recognize taxi detour.

- Monitoring Animal Behavior: By placing wireless sensor devices on animals to study the activity characteristics, animal research institutions obtain activity rule and make good use of traits. Such as before the earthquake, there will be some abnormal animal behavior activity. By analyzing the behavior can be early warning in order to reduce losses. Also, animal scientists study which individual animal leads the group when animals move in order to determine the social hierarchy.

- Privacy and Security: The data mining brings great benefits to us, but at the same time it will inevitably produce the issue of privacy disclosure. It also brings longer-term security threats

- Sports Competition: Data mining techniques have been introduced to competitive sports to improve the athlete's competition results. Through analyzing the reasons and characteristics of the players in different periods, in order to provide some reference for the football match and training. Also, by analyzing the law of the opponent's serve and mining the pattern could prevent opponents score on the court.

- Disease prevention: we could uncover personal mobility patterns and detect abnormal behavior patterns from mobility data obtained with GPS. An abnormal pattern of some patients such as Alzheimer's disease could be detected earlier, will help the families to prevent some dangerous situations in advance [66].

- Health guidelines: With the advent of IT convergence technologies, more people have become aware of improving the quality of human life about smart health services [67]. When a person's figure is saved, the route tracing detects any movement and then traces its location. Analysis on the sequential pattern based biodetection, gives smart health services to determine whether or not the emergency situation is normal.

- Route recommendation: With booming development of tourism industry, understanding the spatial and temporal behavior of visitors could enhance the attraction management and geographical distribution for visitors. To fulfill the need, Tsai et al. [68] defined a Location-Item-Time (LIT) sequence to describe visitor's spatial and temporal behavior. Then, the Location-Item-Time PrefixSpan (LITPrefixSpan) mining algorithm is developed to discover frequentLIT sequential patterns. The recommendation system can provide the customer an efficient moving path and prompt him/ her other popular products to increase cross-selling opportunity.

Based on the practical operation of mobile communication and the development of new technologies, we take a carefully look at data stream technology, moving object technology and LBS based technology. In the future, the application could be extended to more fields using the new technology.

\section{Conclusions}

With the increasing usage of electronic devices, a large amount of spatial-temporal data has been collected in databases. As a result, mining implicit and useful patterns has attracted increasing attention recently and fostered a diversity of applications. In this paper, we highlight several common pattern mining, such as frequent pattern, periodic 
pattern as well as following pattern. It further surveys the applications that are based on patterns mining in some fields. Our paper doesn't presented an in depth analysis of all algorithms which made significant contribution to mining patterns. Also these are more patterns worth detailing. With the growing size of the datasets, development of incremental and distributed patterns mining algorithms has become a necessity.

\section{Acknowledgments}

This work is partially supported by the National Natural Science Foundation of China and the Civil Aviation Administration of China under grant No.U1533133, the National Natural Science Foundation of China under grant No.61002016, the Humanities and Social Sciences Research Project of Ministry of Education of China under grant No.15YJCZH095, Zhejiang Provincial Natural Science Foundation of China under grant No.LY13F010016, Qianjiang Talent Project of Zhejiang Province under grant No.QJD1302014, and the 521 Talents Project of Zhejiang Sci-Tech University.

\section{References}

[1] H. Yuan, Y. Qian, R. Yang and M. Ren, "Human Mobility Discovering and Movement Intention Detection with GPS Trajectories”, Decision Support Systems, vol. 63, (2014), pp. 39-51.

[2] A. Boulmakoul, L. Karim, A. Elbouziri and A. Lbath, "A System Architecture for Heterogeneous Moving-Object Trajectory Metamodel Using Generic Sensors: Tracking Airport Security Case Study”, Systems Journal, IEEE, vol. 9, no. 1, (2015), pp. 283-291.

[3] J. Yuan, Y. Zheng, C. Zhang, W. Xie, X. Xie, G. Sun and Y.Huang, "T-drive: Driving Directions based on Taxi Trajectories", Proceedings of the 18th SIGSPATIAL International conference on advances in geographic information systems, New York, USA, (2010) November 2-5.

[4] C. W. Landsea, and J. L. Franklin, "Atlantic Hurricane Database Uncertainty and Presentation of a New Database Format”, Monthly Weather Review, vol. 141, no. 10, (2013), pp. 3576-3592.

[5] A. Thiebault and Y. Tremblay, "Splitting Animal Trajectories in to Fine-scale Behaviorally Consistent Movement Units: Breaking Points Relate to External Stimuli in A Foraging Seabird", Behavioral Ecology and Sociobiology, vol. 67, no. 6, (2013), pp. 1013-1026.

[6] Q. Shaojie, S. Dayong, W. Xiaoteng, H. N and W. Zhu, "A Self-Adaptive Parameter Selection Trajectory Prediction Approach via Hidden Markov Models", Intelligent Transportation Systems, IEEE Transactions on, vol. 16, no. 1, (2015), pp. 284-296.

[7] F. Wu, T. K. H. Lei, Z. Li, and J. Han, "Movemine 2.0: Mining Object Relationships from Movement Data", Proceedings of the VLDB Endowment, Vol. 7, no. 13, (2014), pp. 1613-1616.

[8] H. Surabi, "Hybrid Model for Intrusion Detection using Data Mining Techniques", Texas A\&M University-Corpus Christi, (2014).

[9] E. H. C. Lu and V.S. Tseng, "Mining Cluster-Based Mobile Sequential Patterns in Location-Based Service Environments", Proceedings of 10th International Conference on Mobile Data Management: Systems, Services and Middleware, Taipei, Taiwan, (2009) May, 18-20.

[10] C. M. Tiu, T. L. Jong and C. W. Hsieh, "Self Organizing Map Neural Network with Fuzzy Screening for Micro-calcifications Detection on Mammograms", Proceedings of 8th IEEE Conference on Soft Computing in Industrial Applications, Muroran, Japan, (2008) June 25-27.

[11] S. U. V. Kumar, "Frequent Pattern Analysis of Moving Objects Using Apriori Algorithm", International Journal of Emerging Research in Management \&Technology, vol.3, no.4, (2014), pp. 139-143.

[12] A. Gomariz, M. Campos, R. Marín and B. Goethals, "Clasp: An Efficient Algorithm for Mining Frequent Closed Sequences", Advances in Knowledge Discovery and Data Mining, Springer, (2013), pp. $50-61$.

[13] K. K. Yip and D.A. Nembhard, "MWASP: Multiple-Width Approximate Sequential Patterns", Computational Intelligence and Data Mining, IEEE Symposium, (2009), pp. 314-319.

[14] I. Tsoukatos, D. Gunopulos, "Efficient Mining of Spatiotemporal Patterns", Springer, (2001).

[15] H. Cao, N. Mamoulis, D.W. Cheung, "Mining Frequent Spatio-temporal Sequential Patterns", Data Mining, 5th IEEE International Conference on IEEE, Houston, USA, (2005) November 27-30.

[16] F. Giannotti, M. Nanni, F. Pinelli and D. Pedreschi, "Trajectory Pattern Mining", Proceedings of the 13th ACM SIGKDD international conference on Knowledge discovery and data mining, New York, USA, (2007) August 12-15.

[17] A. J. Lee, Y. A. Chen and W.-C. Ip, "Mining Frequent Trajectory Patterns in Spatial-temporal Databases", Information Sciences, vol.179, no.13 (2009), pp.2218-2231.

[18] Y. Zhiwen, Y. Zhiyong, Z. Xingshe, C. Becker and Y. Nakamura, "Tree-Based Mining for Discovering Patterns of Human Interaction in Meetings", Knowledge and Data Engineering, vol. 24, no.4, (2012), pp. 759-768. 
[19] C.-C. Hung, W.-C. Peng and W.-C. Lee, "Clustering and aggregating clues of trajectories for mining trajectory patterns and routes", The VLDB Journal-The International Journal on Very Large Data Bases, vol.24, no.2, (2015), pp. 169-192.

[20] G. Gidófalvi and T. B. Pedersen, "Mining Long, Sharable Patterns in Trajectories of Moving Objects", Geoinformatica, vol.13, no.1, (2009), pp.27-55.

[21] V. Colizza, A. Barrat, M. Barthelemy, A. J. Valleron and A. Vespignani, "Modeling the Worldwide Spread of Pandemic Influenza: Baseline Case and Containment Interventions", PLoS medicine, vol.4, no.1, (2007) 95 .

[22] T.-d. Xu, Y. Hao, Z.-r. Peng and L.-j. Sun, "Real-time Travel Time Predictor for Route Guidance Consistent with Driver Behavior", Canadian Journal of Civil Engineering, vol.39, no.10 (2012), pp.1113-1124.

[23] W.-H. Lee, S.-S. Tseng and S. H. Tsai, "A Knowledge Based Real-time Travel Time Prediction System for Urban Network", Expert Systems with Applications, vol.36, no.3, (2009), pp. 4239-4247.

[24] J.H. Faghmous and V. Kumar, "Spatio-temporal Data Mining for Climate Data: Advances, Challenges, and Opportunities", Data Mining and Knowledge Discovery for Big Data, Springer, vol.1, (2014), pp. 83-116.

[25] A. Kitamoto, "Spatio-temporal Data Mining for Typhoon Image Collection", Journal of Intelligent Information Systems, vol.19, no.1, (2002), pp.25-41.

[26] Y. Zheng, F. Liu, H.-P. Hsieh and "U-Air: When Urban Air Quality Inference Meets Big Data", Proceedings of the 19th ACM SIGKDD international conference on Knowledge discovery and data mining, ACM, Chicago, USA, (2013).

[27] Y. Zheng, T. Liu, Y. Wang, Y. Zhu, Y. Liu and E. Chang, "Diagnosing New York City's noises with ubiquitous data", Proceedings of the 2014 ACM International Joint Conference on Pervasive and Ubiquitous Computing, New York, USA, (2014) September 13-17.

[28] S. Su, J. Zhi, L. Lou, F. Huang, X. Chen and J. Wu, "Spatio-temporal Patterns and Source Apportionment of Pollution in Qiantang River (China) Using Neural-based Modeling and Multivariate Statistical Techniques", Physics and Chemistry of the Earth, Parts A/B/C, vol.36, no.9-15, (2011),pp.379-386.

[29] Z. Li, J. Han, B. Ding and R. Kays, "Mining Periodic Behaviors of Object Movements for Animal and Biological Sustainability Studies", Data Mining and Knowledge Discovery, vol.24, no.2, (2012), pp.355386.

[30] Z. Li, M. Ji, J.-G. Lee, L.-A. Tang, Y. Yu, J. Han and R. Kays, "MoveMine: Mining Moving Object Aatabases", Proceedings of the 2010 ACM SIGMOD International Conference on Management of data, Indianapolis, Indiana, (2010) June 6-11.

[31] S. Sarawagi, "Sequence Data Mining", Advanced Methods for Knowledge Discovery from Complex Data, Springer, (2005), pp. 153-187.

[32] Z. Chen, H.T. Shen and X. Zhou, "Discovering Popular Routes from Trajectories", Proceedings of 27th IEEE International Conference on Data Engineering, Washington, USA, (2011) April 11-16.

[33] W. Luo, H. Tan, L. Chen and L.M. Ni, "Finding Time Period-based Most Frequent Path in Big Trajectory Data", Proceedings of the 2013 ACM SIGMOD International Conference on Management of Data, New York, (2013) June 22-27.

[34] G. Liu, H. Zhang and L. Wong, "A Flexible Approach to Finding Representative Pattern Sets, Knowledge and Data Engineering, IEEE Transactions on, vol.26, no.7, (2014), pp. 1562-1574.

[35] Q. Huynh-Thi-Le, T. Le, B. Vo and B. Le, "An Efficient and Effective Algorithm for Mining Top-rank-k Frequent Patterns', Expert Systems with Applications, Vol. 42, no. 1, (2015), pp. 156-164.

[36] R. Y. Zhong, G. Q. Huang, S. Lan, Q. Dai, X. Chen and T. Zhang, "A Big Data Approach for Logistics Trajectory Discovery from RFID-enabled Production Data", International Journal of Production Economics, vol. 165, (2015), pp. 260-272.

[37] F. Rasheeed, M. Alshalalfa and R. Alhajj, "Efficient Periodicity Mining in Time Series Databases Using Suffix Trees", IEEE Transactions on Knowledge and Data Engineering, vol. 23, no. 1,(2010), pp. 79-94.

[38] M.A. Nishi, C.F. Ahmed, M. Samiullah and B.-S. Jeong, "Effective Periodic Pattern Mining in Time Series Databases", Expert Systems with Applications", vol. 40, no. 8, (2013), pp. 3015-3027.

[39] B. Özden, S. Ramaswamy, A. Silberschatz, "Cyclic Association Rules", Proceedings of 14th International Conference on Data Engineering, Orlando, USA, (1998) February 23-27.

[40] M .G. Elfeky, W. G. Aref and A. K. Elmagarmid, "Using Convolution to Mine Obscure Periodic Patterns in One Pass", Advances in Database Technology-EDBT 2004, Springer, (2004).

[41] C. Kim, J. H. Lim, R. T. Ng and K. Shim, "SQUIRE: Sequential Pattern Mining with Quantities", Journal of Systems and Software, vol. 80, no.10, (2007), pp. 1726-1745.

[42] J. Han, W. Gong and Y. Yin, "Mining Segment-Wise Periodic Patterns in Time-Related Databases", KDD, (1998), pp. 214-218.

[43] Y. Xiangzhan and Y. Haining, "An Asynchronous Periodic Sequential Patterns Mining Algorithm with Multiple Minimum Item Supports", Proceedings of 9th International Conference on P2P, Parallel, Grid, Cloud and Internet Computing (3PGCIC), Guangdong, China, (2014) November 8-10. 
[44] Z. Li, B. Ding, J. Han, R. Kays and P. Nye, "Mining Periodic Behaviors for Moving Objects", Proceedings of the 16th ACM SIGKDD international conference on Knowledge discovery and data mining, ACM, Washington, USA, (2010) July 25 - 28.

[45] R. U. Kiran, M. Kitsuregawa and P. K. Reddy, "Efficient Discovery of Periodic-frequent Patterns in Very Large Aatabases", Journal of Systems and Software, vol. 112, (2016), pp. 110-121.

[46] P. Laube and S. Imfeld, "Analyzing Relative Motion within Groups of Trackable Moving Point Objects", Geographic information science, Springer, (2002).

[47] P. Laube, M. van Kreveld and S. Imfeld, "Finding REMO—Detecting Relative Motion Patterns in Geospatial Lifelines", Developments in spatial data handling, Springer, (2005).

[48] P. Kalnis, N. Mamoulis and S. Bakiras, "On Discovering Moving Clusters in Spatio-temporal Data", Advances in spatial and temporal databases, Springer, (2005), pp. 364-381.

[49] Y. Wang, E. P. Lim and S.-Y. Hwang, "Efficient Mining of Group Patterns from User Movement Data", Data \& Knowledge Engineering, vol. 57, nol. 3, (2006), pp. 240-282.

[50] M. Andersson, J. Gudmundsson, P. Laube and T. Wolle, "Reporting Leaders and Followers among Trajectories of Moving Point Objects", GeoInformatica, vol. 12, no. 4, (2007), pp. 497-528.

[51] H. Jeung, M. L. Yiu, X. Zhou, C. S. Jensen and H. T. Shen, "Discovery of Convoys in Trajectory Databases", Proceedings of the VLDB Endowment, vol. 1,no. 1, (2008), pp. 1068-1080.

[52] H. Jeung, H. T. Shen and X. Zhou, "Convoy queries in spatio-temporal databases", Proceedings of IEEE 24th International Conference on Data Engineering, Cancun, Mexico, (2008) April 7-12.

[53] Z. Li, B. Ding, J. Han and R. Kays, "Swarm: Mining relaxed temporal moving object clusters", Proceedings of the VLDB Endowment, vol. 3, no. 1-2, (2010), pp. 723-734.

[54] H. H. Aung and K. L. Tan, "Discovery of evolving convoys", Scientific and Statistical Database Management, Springer, vol. 6187, (2010), pp. 196-213.

[55] F. de Lucca Siqueira and V. Bogorny, "Discovering Chasing Behavior in Moving Object Trajectories", Transactions in GIS, vol. 15, no.5, (2011), pp. 667-688.

[56] L.-A. Tang, Y. Zheng, J. Yuan, J. Han, A. Leung, C.-C. Hung and W.-C. Peng, "On Discovery of Traveling Companions from Streaming Trajectories", Proceedings of IEEE 28th International Conference on Data Engineering (ICDE), Washington, USA, (2012) April 1-5.

[57] L. Zhenhui, W. Fei and M. C. Crofoot, "Mining Following Relationships in Movement Data", Proceedings of IEEE 13th International Conference on Data Mining (ICDM), Dallas, USA, (2013) December 7-10.

[58] U. Turdukulov, A.O. Calderon Romero, O. Huisman and V. Retsios, "Visual Mining of Moving Flock Patterns in Large Spatio-temporal Data Sets Using A Frequent Pattern Approach", International Journal of Geographical Information Science, vol. 28, no.10, (2014), pp. 2013-2029.

[59] P. Laube, S. Imfeld and R. Weibel, "Discovering relative motion patterns in groups of moving point objects", International Journal of Geographical Information Science, vol. 19,no. 6, (2005), pp. 639-668.

[60] Z. Li, B. Ding, F. Wu, T.K.H. Lei, R. Kays and M .C. Crofoot, "Attraction and Avoidance Detection from Movements", Proceedings of the VLDB Endowment, vol. 7, no.3, (2013) pp. 157-168.

[61] F. Orakzai, T. Devogele and T. Calders, "Towards Distributed Convoy Pattern Mining", Computer Science, (2015).

[62] Y. Li, J. Bailey and L. Kulik, "Efficient Mining of Platoon Patterns in Trajectory Databases", Data \& Knowledge Engineering, vol. 100, (2015), pp. 167-187.

[63] R. Trasarti, A. M. Olteanu-Raimond, M. Nanni, T. Couronné, B. Furletti, F. Giannotti, Z. Smoreda and C. Ziemlicki, "Discovering Urban and Country Dynamics from Mobile Phone Data with Spatial Correlation Patterns", Telecommunications Policy, vol. 39, no. 3-4, (2015), pp. 347-362.

[64] W. Qiu and A. Bandara, "GPS Trace Mining for Discovering Behavior Patterns", 2015 International Conference on Intelligent Environments (IE), IEEE, Prague, Czech (2015) July 15-17.

[65] D. Zhang, N. Li, Z.-H. Zhou, C. Chen, L. Sun and S. Li, "iBAT: Detecting Anomalous Taxi Trajectories from GPS Traces", Proceedings of the 13th international conference on Ubiquitous computing, ACM, Beijing, China, (2011) September 17-21.

[66] D. H. Shih, M. H. Shih, D. C. Yen and J. H. Hsu, "Personal Mobility Pattern Mining and Anomaly Detection in the GPS Era", Cartography and Geographic Information Science, vol. 43, no.1, (2016), pp.55-67.

[67] H. Jung and K. Chung, "Sequential Pattern Profiling based Bio-detection for Smart Health Service", Cluster Computing, vol.18, no.1, (2015), pp. 209-219.

[68] C. Y. Tsai and B. H. Lai, "A location-item-time Sequential Pattern Mining Algorithm for Route Recommendation”, Knowledge-Based Systems, vol.73, (2015), pp. 97-110. 


\begin{abstract}
Authors
Li Chen is a postgraduate student in Zhejiang Sci-Tech University. Her main research fields are data mining, data exploration, Knowledge Discovery.

Lianggui Liu received the $\mathrm{PhD}$ degrees in Communications and information system from Nanjing University of Posts \& Telecommunications, Nanjing, China. $\mathrm{He}$ is now an associate professor with the School of Information Science and Technology, Zhejiang Sci-Tech University, Hangzhou, China. His current research interests focus on network communications, network security and natural computation.
\end{abstract}
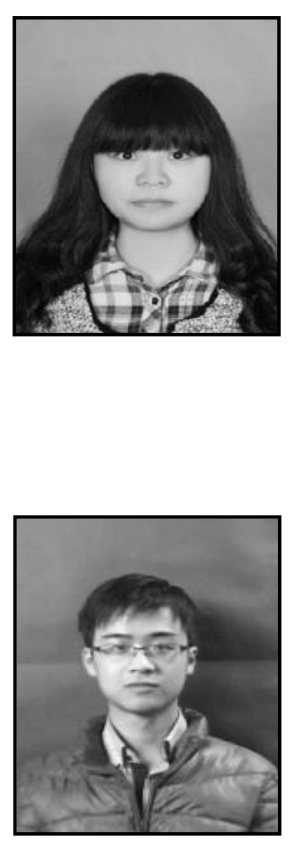

Bingxian Chen is a postgraduate student in Zhejiang Sci-Tech University. His main research fields are network communications, network security.

Huiling Jia received the $\mathrm{PhD}$ degrees in Communication and Information System from Zhejiang University. She is now a lecturer with School of Information, Zhejiang Sci-Tech University, Hangzhou, China. Her current research interests include wireless sensor networks, heterogeneous wireless networks, radio resource management.

Yu Zhang is now an associate professor with the School of Information Science and Technology, Zhejiang Sci-Tech University, Hangzhou, China. His current research interests focus on trusted computing, social network analysis and Web data mining and knowledge discovery. 